\title{
Incorporation field crop residues in rabbit rations
}

\author{
Hamed A. A. Omer ${ }^{1}$, Mohamed F. El Karamany², Sawsan M. Ahmed', Soha S. Abdel-Magid", Soad El-Naggar ${ }^{1}$ \\ and Bakry A. Bakry²
}

\begin{abstract}
Background: In Egypt, the area of Berseem was decreased and wheat area increased which lead to high price of rabbit rations. Using non-traditional feed led to decrease the cost of feeding. The main objective of this study is to replace $50 \%$ of Berseem hay $(\mathrm{BH})$ of rabbit rations by agriculture by-products such as Mung bean husks (MBH), soybean vein hay $(\mathrm{SBVH})$, or peanut vein hay $(\mathrm{PVH})$.

Method: Twenty-four New Zealand White (NZW) rabbits aged 5-6 weeks (584 $\pm 25 \mathrm{~g}$ ) were randomly divided into four equal experimental groups. Feeding trial lasted 70 days.

Results: Except for organic matter (OM) digestibility, treatments had no effect on all other nutrient digestibilites that includes dry matter (DM), crude protein (CP), crude fiber (CF), ether extract (EE), and nitrogen free extract (NFE) and nutritive values \{total digestible nutrient (TDN) and digestible crude protein (DCP)\}. Instead, 50\% of BH by the other alternative source of agriculture by-products had no effect on all carcass parameters measured. However, dressing percentages was improved. Treatments had no effect on albumin, total lipids, triglycerides, total cholesterol, low-density lipoprotein (LDL), glutamic oxaloacetic transaminase (GOT), creatinine, and alkaline phosphatase.

Conclusion: It can be instead 50\% of hay used in rabbit rations with alternative sources of roughage, such as Mung bean husks, soybean vein hay, or peanut vein hay, without any adverse effect on their performance, digestion, carcass characteristics, and blood constituents.
\end{abstract}

Keywords: Berseem hay, Agriculture by-products, Carcass characteristics, Blood constituents

\section{Background}

One of the very important limiting factors for animal production in Egypt is the availability of feed stuffs. Locally produced feeds are not sufficient to cover the nutritional requirements of livestock (Abou-Akkada 1988).

In Egypt, the total area planted by clover hay reached about two million feddans (EMA 2003); recently according to the national policy, the area of Berseem was decreased and wheat area increased which lead to high price of rabbit rations (Abo EL-Maaty et al. 2014). Using non-traditional feed led to decrease the cost of feeding and alleviate the pollution problems (Abdel-Magid Soha et al. 2008).

The volume of agriculture by-products reached 35 million tons annually, 23 million tons of which were

\footnotetext{
* Correspondence: Soha_Syd@yahoo.com

${ }^{1}$ Animal Production Department, National Research Centre, 33 El-Bohouth

Street, P.O: 12622, Dokki, Cairo, Egypt

Full list of author information is available at the end of the article
}

plant wastes (7 million tons of them are used as fodders; 4 million tons were used as organic fertilizers; and the rest of wastes which 12 million tons were left without any use) as noted by Sadek Enath (2013).

Recently, some studies were designed to use some agricultural by-products in rabbit feeding, especially as alternatives to Berseem hay, which commonly represents about $30-40 \%$ of the complete pelleted diets of rabbits. Peanut and Mung beans and kidney beans are cultivated in the newly reclaimed lands. So, significant amounts of their straws and by-products of these crops are produced annually that may help in the solution of the shortage of animal feeding (Omer et al. 2012).

Rabbits have fast reproductive and growth rates and are excellent species in converting feed into body weight. They are both known to yield high-quality protein meat with low fat; rabbits have a small body size but can be raised on relatively small amounts of non-conventional 
feedstuffs Cheeke (1986). They can be produced on grain-free diets, mainly on forages and other type of agricultural by-products. The specific advantages of rabbits have been reviewed by Cheeke et al. (1987); some of these advantages make rabbits a suitable livestock species for meat production in the developing nations.

So, this work aimed to investigate the influence of replacing $50 \%$ of Berseem hay that is considered the main source of roughage in rabbit rations with untraditional sources of roughage (Mung bean husks, soybean vein hay, and peanut vein hay) on rabbit's performance, digestion coefficients, nutritive values, carcass characteristics, and blood constituents.

\section{Methods}

The present study was carried out at El-Nubaria Experimental and Production Station at El-Imam Malik Village.

\section{Experimental animals and feeds}

From September to November, 2017, 24 New Zealand White (NZW) rabbits aged 5-6 weeks with an average body weight of $584 \pm 25 \mathrm{~g}$ were randomly divided into four equal experimental groups (6 rabbits in each treatment).

Rabbits were housed in galvanized wire cages $(50 \times 50 \times$ $45 \mathrm{~cm}$ ) and provided with stainless steel nipples for drinking and feeders allowing recording feed intake during the feeding trial that continued for 70 days.

All experimental group rabbits were kept under the same managerial conditions, and rations were offered pelleted with diameter $4 \mathrm{~mm}$.

The experimental pelleted rations were formulated to cover the nutrient requirements for rabbits according to NRC (1977) as described in Tables 1 and 2. Rabbits received one of the fourth rations as follows:

The first one was considered as control $\left(\mathrm{R}_{1}\right)$. The second, third, and fourth experimental rations were formulated by replacing $50 \%$ of Berseem hay $(\mathrm{BH})$ by alternative sources of roughage such as $\mathrm{MBH}, \mathrm{SBVH}$, and $\mathrm{PVH}$, for $\mathrm{R}_{2}, \mathrm{R}_{3}$, and $\mathrm{R}_{4}$, respectively. Rations were offered ad libitum.

\section{Digestibility trials}

At the end of the feeding trial, three rabbits from each treatment were used in a digestibility trial that carried out over a period of 7 days. Feces were daily collected quantitatively during the collection period before feeding at 8:30 a.m. Feed intake of experimental rations and weight of feces were also daily recorded. Representative samples of $10 \%$ of total fresh weight of feces were sprayed with solution of $10 \%$ sulfuric acid and 10\% formaldehyde and oven dried at $60{ }^{\circ} \mathrm{C}$ for $48 \mathrm{~h}$, and composite samples of dried feces were ground and stored for later chemical analysis. The nutritive values expressed TDN and DCP of experimental rations that were calculated using a classic method as described by Abou-Raya (1967).
Table 1 Composition of the experimental rations

\begin{tabular}{lllll}
\hline Item & $\mathrm{BH}$ & $\mathrm{MBH}$ & $\mathrm{SBVH}$ & $\mathrm{PVH}$ \\
& $\left(\mathrm{R}_{1}\right)$ & $\left(\mathrm{R}_{2}\right)$ & $\left(\mathrm{R}_{3}\right)$ & $\left(\mathrm{R}_{4}\right)$ \\
\hline Yellow corn & 150 & 130 & 150 & 150 \\
Soybean meal & 240 & 260 & 240 & 240 \\
Wheat bran & 170 & 170 & 170 & 170 \\
Barley grain & 180 & 180 & 180 & 180 \\
Berseem hay & 230 & 115 & 115 & 115 \\
Mung bean husks & - & 115 & - & - \\
Soybean vein hay & - & - & 115 & - \\
Peanut vein hay & - & - & - & 115 \\
Di-calcium & 12 & 12 & 12 & 12 \\
Limestone $^{\text {Sodium chloride }}$ & 12 & 12 & 12 & 12 \\
Vit. and Min. mixture $^{1}$ & 3 & 3 & 3 & 3 \\
D-L-Methionine & 1 & 2 & 2 & 2 \\
\hline Vit. and Min. mixtur: & 2 & 1 & 1 & 1
\end{tabular}

${ }^{1}$ Vit. and Min. mixture: each kilogram of Vit. and Min. mixture contains 2000.000 IU Vit. A, 150.000 IU Vita. D, $8.33 \mathrm{~g}$ Vit. E, $0.33 \mathrm{~g}$ Vit. $K, 0.33 \mathrm{~g}$ Vit. $B_{1}$, $1.0 \mathrm{~g}$ Vit. $B_{2}, 0.33 \mathrm{~g}$ Vit. $B_{6}, 8.33 \mathrm{~g}$ Vit. $B_{5}, 1.7 \mathrm{mg}$ Vit. $B 1_{2}, 3.33 \mathrm{~g}$ pantothenic acid, $33 \mathrm{mg}$ biotin, $0.83 \mathrm{~g}$ folic acid, $200 \mathrm{~g}$ choline chloride, $11.7 \mathrm{~g} \mathrm{Zn}, 12.5 \mathrm{~g}$ $\mathrm{Fe}, 16.6 \mathrm{mg} \mathrm{Se}, 16.6 \mathrm{mg} \mathrm{Co}, 66.7 \mathrm{~g} \mathrm{Mg}$, and $5 \mathrm{~g} \mathrm{Mn}$

$B H$ Berseem hay, MBH Mung bean husks, SBVH soybean vein hay, $P V H$ peanut vein hay

\section{Slaughter trials}

Three representative rabbits from each treatment were randomly chosen to determine the carcass parameters according to Blasco et al. 1993. Rabbits were fasted for $12 \mathrm{~h}$ before slaughter, which was performed according to the Islamic rules. Animals were weighed just before slaughter, slaughter weight $(\mathrm{SW})$ was recorded and as well as after complete bleeding.

Total edible offals (Giblets) which included heart, liver, kidneys, lungs, spleen, and testes were weighed.

Full and empty weights of digestive tract were recorded. Hot carcass which included head and giblets were weighed to calculate dressing percentages.

\section{Blood sample collection and analytical procedures}

Blood samples were collected from the slaughtered rabbits (three of each treatment) and centrifuged at 4000 r.p.m. for $20 \mathrm{~min}$, for the preparation of blood plasma. Plasma kept frozen at $-18{ }^{\circ} \mathrm{C}$ for subsequent analysis. Plasma total protein was determined according to Armstrong and Carr (1964) and Witt and Trendelenburg (1982); albumin was determined according to Doumas et al. (1971) and Tietz (1986); triglycerides were determined according to Fossati and Principe (1982); total lipids were determined according to Postma and Stroes (1968)); total cholesterol was determined according to Allain et al. (1974) and Pisani et al. (1995); alkaline phosphates' activity was measured according to the method of Beliefield and Goldberg (1971); plasma 
Table 2 Chemical analysis of the experimental rations

\begin{tabular}{lllll}
\hline Item & $\mathrm{BH}\left(\mathrm{R}_{1}\right)$ & $\mathrm{MBH}\left(\mathrm{R}_{2}\right)$ & $\mathrm{SBVH}\left(\mathrm{R}_{3}\right)$ & $\mathrm{PVH}\left(\mathrm{R}_{4}\right)$ \\
\hline Moisture & 9.12 & 9.15 & 9.33 & 9.40 \\
$\begin{array}{l}\text { Chemical analysis (\%) } \\
\text { on DM basis }\end{array}$ & & & & \\
OM & 89.42 & 89.69 & 89.40 & 89.51 \\
CP & 20.89 & 20.79 & 20.69 & 20.74 \\
CF & 12.17 & 12.38 & 14.59 & 13.22 \\
EE & 2.77 & 2.45 & 2.77 & 2.84 \\
NFE & 53.59 & 54.07 & 51.35 & 52.71 \\
Ash & 10.58 & 10.31 & 10.60 & 10.49 \\
Neutral detergent fiber NDF) & 36.39 & 36.74 & 37.03 & 37.84 \\
Gross energy (kcal/kg DM) & 4170 & 4163 & 4166 & 4175 \\
$\begin{array}{l}\text { Digestible energy (Mcal/kg } \\
\text { DM) }\end{array}$ & 2.577 & 2.560 & 2.546 & 2.506 \\
$\begin{array}{l}\text { Non fibrous carbohydrates } \\
\text { (NFC) }\end{array}$ & 29.37 & 29.71 & 28.91 & 28.09 \\
\hline
\end{tabular}

Gross energy (kcal/kg DM) was calculated (Blaxter 1968)

Digestible energy (M cal) was calculated (Cheeke 1987)

$B H$ Berseem hay, $M B H$ Mung bean husks, SBVH soybean vein hay, $P V H$ peanut vein hay, NFC non-fibrous carbohydrates were calculated (Calsamiglia et al. 1995)

glutamic oxaloacetic transaminase (GOT) and glutamic pyruvic transaminase (GPT) activities were determined as described by Reitman and Frankel (1957) and Harold (1975); creatinine was estimated according to Husdan (1968)); high-density lipoprotein (HDL) was estimated according to Assmann (1979); and low-density lipoprotein (LDL) was determined as method described by McNamara et al. (1990) using commercial kits. On the other hand, globulin and albumin to globulin ratio (A:G ratio) were calculated.

Chemical analyses of experimental rations and feces were analyzed according to AOAC (2005) methods. Meanwhile, neutral detergent fiber (NDF) was determined according to Goering and Van Soest (1970) and Van Soest et al. (1991).

\section{Calculations}

Gross energy ( $\mathrm{kcal} / \mathrm{kg} \mathrm{DM})$ was calculated according to Blaxter (1968). Each g CP $=5.65 \mathrm{Kcal}, \mathrm{g} \mathrm{EE}=9.40 \mathrm{kcal}$ and $\mathrm{g}(\mathrm{CF}$ and NFE $)=4.15 \mathrm{kcal}$.

Digestible energy (DE) was calculated according to Cheeke (1987) by applying the following equation: DE $(\mathrm{Mcal} / \mathrm{kg} \mathrm{DM})=4.36-0.049 \times \mathrm{NDF}$.

Non-fibrous carbohydrates (NFC), calculated according to Calsamiglia et al. (1995) using the following equation: $\mathrm{NFC}=100-\{\mathrm{CP}+\mathrm{EE}+\mathrm{Ash}+\mathrm{NDF}\}$.

\section{Statistical analysis}

Data collected of feed intake, live body weight; feed conversion, nutrient digestibility, blood constituents, and carcass data were subjected to statistical analysis as one-way analysis of variance according to SPSS (2008). Duncan's multiple range test (Duncan 1955) was used to separate means when the dietary treatment effect was significant according to the following model:

$$
\mathrm{Yij}=\mu+\mathrm{Ti}+\mathrm{eij}
$$

where

$Y_{\mathrm{ij}}=$ observation

$\mu=$ overall mean

$\mathrm{T}_{\mathrm{i}}=$ effect of experimental rations for $i=1-4,1=$ (control ration contained $23 \% \mathrm{BH}$ ), $2=$ replacing $50 \%$ of $\mathrm{BH}$ with $\mathrm{MBH}$

$3=$ replacing $50 \%$ of $\mathrm{BH}$ with $\mathrm{SBVH}$ and

$4=$ replacing $50 \%$ of $\mathrm{BH}$ in with $\mathrm{PVH}$

$\mathrm{e}_{\mathrm{ij}}=$ the experimental error.

\section{Results}

\section{Chemical analysis}

Experimental rations were almost iso-caloric (ranged from 4163 to $4175 \mathrm{kcal} / \mathrm{kg} \mathrm{DM}$ ) and also iso nitrogenous (ranged from 20.69 to $20.89 \% \mathrm{CP}$ ). Meanwhile, approximately, EE content was in the same range that ranged from 2.45 to $2.84 \%$ among the four experimental tested rations. On the other hand, CF content was varied from 12.17 to $14.59 \%$; this is related to the differences in tested materials content of CF. Also, NDF content was slightly increased with instead $50 \%$ of $\mathrm{BH}$ in control ration by the three other tested materials used in ration formulation. Digestible energy contents were slightly decreased for tested rations compared to the control Table 2.

\section{Nutrient digestibility and nutritive values}

Data illustrated in Table 3 mentioned that, except OM digestibility, treatments had no significant effect on all other nutrient digestibilites and nutritive values. However, replacing $50 \%$ of Berseem hay in control ration with tested materials slightly improved both nutrient digestibility and nutritive values for experimental rations $\left(R_{2}\right.$ recorded the best values) so it can be safely used as untraditional source of roughage or as instead of Berseem hay.

\section{Growth performance}

Replacing $50 \%$ of $\mathrm{BH}$ with $\mathrm{MBH}, \mathrm{SBVH}$, or $\mathrm{PVH}$ (Table 4) improved FW, TBWG, ADG, and feed conversion that expressed as (g DMI/g gain). However, no significant decrease occurred with daily dry matter intake.

\section{Carcass characteristics Dressing percentages}

Treatments had no significant effect on all carcass parameters measured including SW, DT, EBW, giblets, DP, and carcass cuts (Table 5). 
Table 3 Nutrient digestibility and nutritive values of the experimental rations

\begin{tabular}{|c|c|c|c|c|c|}
\hline Item & $\mathrm{BH}\left(\mathrm{R}_{1}\right)$ & $\mathrm{MBH}\left(\mathrm{R}_{2}\right)$ & $\mathrm{SBVH}\left(\mathrm{R}_{3}\right)$ & $\mathrm{PVH}\left(\mathrm{R}_{4}\right)$ & SEM \\
\hline \multicolumn{6}{|c|}{ Digestion coefficients (\%) } \\
\hline DM & 88.16 & 89.21 & 89.14 & 88.32 & 0.50 \\
\hline $\mathrm{OM}$ & $71.23^{b}$ & $75.14^{\mathrm{a}}$ & $74.26^{\mathrm{a}}$ & $72.94^{\mathrm{ab}}$ & 0.58 \\
\hline $\mathrm{CP}$ & 63.25 & 65.43 & 64.17 & 63.80 & 0.49 \\
\hline CF & 56.11 & 58.12 & 57.29 & 56.33 & 0.41 \\
\hline $\mathrm{EE}$ & 63.22 & 66.13 & 65.86 & 64.88 & 0.53 \\
\hline NFE & 77.66 & 79.21 & 78.83 & 77.75 & 0.50 \\
\hline \multicolumn{6}{|c|}{ Nutritive values (\%) } \\
\hline TDN & 65.60 & 67.27 & 66.22 & 65.81 & 0.39 \\
\hline DCP & 13.21 & 13.60 & 13.28 & 13.23 & 0.10 \\
\hline
\end{tabular}

\section{External and internal offals}

Treatments had no effect on both total external and internal offals (Table 6) that expressed as weight or \% of slaughter weight.

\section{Digestive tract weights and length}

Treatments had no significant effect on the full and empty weight of the stomach, large intestine, and total digestive tract and the length of the stomach and small and large intestine. Also, present results mentioned that stomach length was slightly increased, while small intestine length decreased with replacing $\mathrm{BH}$ with $(\mathrm{MBH}, \mathrm{SBVH}$, or $\mathrm{PVH})$. However, except $\mathrm{R}_{2}$, the large intestine length was insignificantly decreased when rabbits received diets that were replaced by $50 \%$ of Berseem hay in control ration by $\mathrm{SBVH}$ or PVH (Table 7).

Table 4 Growth performance of the experimental groups

\begin{tabular}{llllll}
\hline Item & $\mathrm{BH}\left(\mathrm{R}_{1}\right)$ & $\mathrm{MBH}\left(\mathrm{R}_{2}\right)$ & $\mathrm{SBVH}\left(\mathrm{R}_{3}\right)$ & $\mathrm{PVH}\left(\mathrm{R}_{4}\right)$ & $\mathrm{SEM}$ \\
\hline Initial weight (g) & 584 & 580 & 588 & 582 & 24.88 \\
Final weight (FW, g) & 2041 & 2321 & 2206 & 2186 & 70.50 \\
$\begin{array}{l}\text { Total body weight } \\
\text { gain (TBWG, g) }\end{array}$ & 1457 & 1741 & 1618 & 1604 & 73.10 \\
$\begin{array}{l}\text { Average daily gain } \\
\text { (ADG, g/day) }\end{array}$ & 20.81 & 24.87 & 23.11 & 22.91 & 1.04 \\
$\begin{array}{l}\text { Daily dry matter } \\
\text { (DMI), g }\end{array}$ & 119.1 & 114.4 & 109.5 & 112.3 & 4.20 \\
$\begin{array}{l}\text { Feed conversion } \\
\text { (g DMI/g gain) }\end{array}$ & 5.72 & 4.60 & 4.74 & 4.90 & 0.20 \\
\hline
\end{tabular}

SEM standard error of mean, $B H$ Berseem hay, $M B H$ Mung bean husks, SBVH soybean vein hay, $P V H$ peanut vein hay
Table 5 Dressing percentages and carcass cuts of the experimental groups

\begin{tabular}{|c|c|c|c|c|c|}
\hline Item & $\mathrm{BH}\left(\mathrm{R}_{1}\right)$ & $\mathrm{MBH}\left(\mathrm{R}_{2}\right)$ & $\mathrm{SBVH}\left(\mathrm{R}_{3}\right)$ & $\mathrm{PVH}\left(\mathrm{R}_{4}\right)$ & SEM \\
\hline Slaughter weight (SW), g & 2451 & 2188 & 2353 & 2169 & 91.94 \\
\hline \multicolumn{6}{|l|}{ Digestive tract (DT) } \\
\hline Full weight, g & 420 & 331 & 376 & 326 & 18.65 \\
\hline Empty weight, g & 166 & 162 & 151 & 181 & 6.44 \\
\hline Content weight, g & $254^{a}$ & $169^{\mathrm{ab}}$ & $225^{a b}$ & $145^{b}$ & 18.99 \\
\hline $\begin{array}{l}\text { Empty body weight } \\
\text { (EBW), g }\end{array}$ & 2197 & 2019 & 2128 & 2024 & 80.08 \\
\hline Head weight, g & 125 & 120 & 117 & 124 & 3.46 \\
\hline $\begin{array}{l}\text { Edible offal's (giblets) } \\
\text { weight, } g\end{array}$ & 141 & 112 & 138 & 116 & 6.27 \\
\hline Carcass weight $\left(\mathrm{CW}_{1}\right)$ & 1256 & 1165 & 1231 & 1145 & 54.61 \\
\hline Carcass weight $\left(\mathrm{CW}_{2}\right)$ & 1381 & 1285 & 1348 & 1269 & 57.80 \\
\hline Carcass weight $\left(\mathrm{CW}_{3}\right)$ & 1522 & 1397 & 1486 & 1385 & 63.38 \\
\hline \multicolumn{6}{|l|}{$\begin{array}{l}\text { Dressing percentages } \\
\text { (DP)\% }\end{array}$} \\
\hline $\mathrm{DP}^{1}$ & 51.24 & 53.24 & 52.32 & 52.79 & 0.65 \\
\hline $\mathrm{DP}^{2}$ & 56.34 & 58.73 & 57.29 & 58.51 & 0.62 \\
\hline $\mathrm{DP}^{3}$ & 62.10 & 63.85 & 63.15 & 63.85 & 0.59 \\
\hline $\mathrm{DP}^{4}$ & 57.17 & 57.70 & 57.84 & 56.57 & 0.59 \\
\hline $\mathrm{DP}^{5}$ & 62.86 & 63.64 & 63.35 & 62.70 & 0.58 \\
\hline $\mathrm{DP}^{6}$ & 69.28 & 69.19 & 69.83 & 68.43 & 0.63 \\
\hline
\end{tabular}

Carcass cuts (g or \%

of $\left(W_{1}\right)$

\begin{tabular}{llllll} 
Fore limbs & 410 & 448 & 392 & 376 & 20.99 \\
& $32.64^{\mathrm{b}}$ & $38.45^{\mathrm{a}}$ & $31.84^{\mathrm{b}}$ & $32.84^{\mathrm{b}}$ & 0.99 \\
Loin & 374 & 303 & 354 & 309 & 19.07 \\
& 29.78 & 26.01 & 28.76 & 26.99 & 0.67 \\
Hind limbs & 472 & 414 & 485 & 460 & 18.01 \\
& 37.58 & 35.54 & 39.40 & 40.17 & 0.82 \\
\hline
\end{tabular}

$\mathrm{a}$ and $\mathrm{b}$ : means in the same row having different superscripts differ significantly $(P<0.05)$

SEM standard error of mean, $B H$ Berseem hay, $M B H$ Mung bean husks, SBVH soybean vein hay, $\mathrm{PVH}$ peanut vein hay

EBW: Empty body weight $=$ Slaughter weight - digestive tract content $\mathrm{CW}_{1}$ : Carcass weight

$\mathrm{CW}_{2}$ : Carcass weight + head

$\mathrm{CW}_{3}$ : Carcass weight + head + edible offals included (liver, heart, kidneys, lungs, testes, and spleen)

$\mathrm{DP}_{1}$ : Dressing percentages calculated as $\left(\mathrm{CW}_{1} / \mathrm{SW} \times 100\right)$ $\mathrm{DP}_{2}$ : Dressing percentages calculated as $\left(\mathrm{CW}_{2} / \mathrm{SW} \times 100\right)$ $\mathrm{DP}_{3}$ : Dressing percentages calculated as $\left(\mathrm{CW}_{3}+\mathrm{SW} \times 100\right)$ $\mathrm{DP}_{4}$ : Dressing percentages calculated as $\left(\mathrm{CW}_{1} / \mathrm{EBW} \times 100\right)$ $\mathrm{DP}_{5}$ : Dressing percentages calculated as $\left(\mathrm{CW}_{2} / \mathrm{EBW} \times 100\right)$

$\mathrm{DP}_{6}$ : Dressing percentages calculated as $\left(\mathrm{CW}_{3}+\mathrm{EBW} \times 100\right)$

\section{Blood parameters}

Treatments had no effect on albumin, total lipids, triglycerides, total cholesterol, low-density lipoprotein, GOT, creatinine, and alkaline phosphatase (Table 8). Total protein and globulin $\left(\mathrm{R}_{3}\right)$ and HDL $\left(\mathrm{R}_{2}\right)$ increased $(P<0.05)$, while GPT $\left(R_{3}\right)$ decreased $(P<0.05)$ compared to control. 
Table 6 External and internal offal's (Giblets) of the experimental groups

\begin{tabular}{|c|c|c|c|c|c|}
\hline Item & $\mathrm{BH}\left(\mathrm{R}_{1}\right)$ & $\mathrm{MBH}\left(\mathrm{R}_{2}\right)$ & $\mathrm{SBVH}\left(\mathrm{R}_{3}\right)$ & $\mathrm{PVH}\left(\mathrm{R}_{4}\right)$ & SEM \\
\hline Slaughter weight (SW), g & 2451 & 2188 & 2353 & 2169 & 91.94 \\
\hline \multicolumn{6}{|l|}{$\begin{array}{l}\text { External offal's (g or \% } \\
\text { of SW) }\end{array}$} \\
\hline Blood & $\begin{array}{l}49 \\
2.00\end{array}$ & $\begin{array}{l}54 \\
2.47\end{array}$ & $\begin{array}{l}52 \\
2.21\end{array}$ & $\begin{array}{l}57 \\
2.63\end{array}$ & $\begin{array}{l}2.39 \\
0.12\end{array}$ \\
\hline Fur, legs, ears and tail & $\begin{array}{l}459 \\
18.73\end{array}$ & $\begin{array}{l}406 \\
18.56\end{array}$ & $\begin{array}{l}438 \\
18.61\end{array}$ & $\begin{array}{l}402 \\
18.53\end{array}$ & $\begin{array}{l}19.03 \\
0.34\end{array}$ \\
\hline Total external offals & $\begin{array}{l}508 \\
20.73\end{array}$ & $\begin{array}{l}460 \\
21.03\end{array}$ & $\begin{array}{l}490 \\
20.82\end{array}$ & $\begin{array}{l}459 \\
21.16\end{array}$ & $\begin{array}{l}19.79 \\
0.36\end{array}$ \\
\hline \multicolumn{6}{|l|}{$\begin{array}{l}\text { Edible offals (g or } \% \text { of } \\
\text { SW) }\end{array}$} \\
\hline Head & $\begin{array}{l}125 \\
5.10^{\mathrm{bc}}\end{array}$ & $\begin{array}{l}120 \\
5.48^{\mathrm{ab}}\end{array}$ & $\begin{array}{l}117 \\
4.97^{c}\end{array}$ & $\begin{array}{l}124 \\
5.72^{\mathrm{a}}\end{array}$ & $\begin{array}{l}3.46 \\
0.11\end{array}$ \\
\hline \multicolumn{6}{|l|}{$\begin{array}{l}\text { Internal offal's (giblets), } \\
\text { (g or \% of SW) }\end{array}$} \\
\hline Liver & $\begin{array}{l}84^{\mathrm{a}} \\
3.43^{\mathrm{a}}\end{array}$ & $\begin{array}{l}58^{\mathrm{b}} \\
2.65^{\mathrm{b}}\end{array}$ & $\begin{array}{l}82^{\mathrm{a}} \\
3.48^{\mathrm{a}}\end{array}$ & $\begin{array}{l}62^{\mathrm{ab}} \\
2.86^{\mathrm{b}}\end{array}$ & $\begin{array}{l}4.58 \\
0.11\end{array}$ \\
\hline Heart & $\begin{array}{l}9 \\
0.37\end{array}$ & $\begin{array}{l}10 \\
0.46\end{array}$ & $\begin{array}{l}11 \\
0.47\end{array}$ & $\begin{array}{l}7 \\
0.32\end{array}$ & $\begin{array}{l}0.73 \\
0.03\end{array}$ \\
\hline Kidneys & $\begin{array}{l}23 \\
0.94^{\mathrm{ab}}\end{array}$ & $\begin{array}{l}22 \\
1.01^{\mathrm{a}}\end{array}$ & $\begin{array}{l}19 \\
0.81^{\mathrm{b}}\end{array}$ & $\begin{array}{l}20 \\
0.92^{\mathrm{ab}}\end{array}$ & $\begin{array}{l}1.36 \\
0.03\end{array}$ \\
\hline Lungs & $\begin{array}{l}16 \\
0.65\end{array}$ & $\begin{array}{l}12 \\
0.55\end{array}$ & $\begin{array}{l}14 \\
0.59\end{array}$ & $\begin{array}{l}15 \\
0.69\end{array}$ & $\begin{array}{l}0.78 \\
0.03\end{array}$ \\
\hline Testes & $\begin{array}{l}9 \\
0.37^{b}\end{array}$ & $\begin{array}{l}10 \\
0.46^{\mathrm{a}}\end{array}$ & $\begin{array}{l}10 \\
0.42^{\mathrm{ab}}\end{array}$ & $\begin{array}{l}10 \\
0.46^{\mathrm{a}}\end{array}$ & $\begin{array}{l}0.28 \\
0.01\end{array}$ \\
\hline Spleen & $\begin{array}{l}1 \\
0.04\end{array}$ & $\begin{array}{l}1 \\
0.05\end{array}$ & $\begin{array}{l}1 \\
0.04\end{array}$ & $\begin{array}{l}1 \\
0.05\end{array}$ & $\begin{array}{l}0.00 \\
0.02\end{array}$ \\
\hline $\begin{array}{l}\text { Total internal offals } \\
\text { (giblets) }\end{array}$ & $\begin{array}{l}141 \\
5.75\end{array}$ & $\begin{array}{l}112 \\
5.12\end{array}$ & $\begin{array}{l}138 \\
5.86\end{array}$ & $\begin{array}{l}116 \\
5.35\end{array}$ & $\begin{array}{l}6.27 \\
0.10\end{array}$ \\
\hline
\end{tabular}

$a, b$, and $c$ : means in the same row having different superscripts differ significantly $(P<0.05)$

SEM standard error of mean, $B H$ Berseem hay, $M B H$ Mung bean husks, SBVH soybean vein hay, $\mathrm{PVH}$ peanut vein hay

\section{Discussion}

The main objective of this study was to investigate the influence of replacing $50 \%$ of $\mathrm{BH}$ of rabbit ration formulation by alternative sources of agriculture by-products such as $\mathrm{MBH}, \mathrm{SBVH}$, or $\mathrm{PVH}$. The present results of Table 3 in agreement with those found by Asar et al. (2010) who noted that digestion coefficients of (DM, $\mathrm{OM}, \mathrm{CP}, \mathrm{EE}$, and NFE), TDN and DCP were improved by replacing barley with alfalfa hay, Berseem hay, or dried Faba bean straw each at $25 \%$ as a fiber source for rabbits fed rations contained 30\% corn-cob meal. On the other hand, Ghazalah and El-Shahat (1994) showed that inclusion of olive meal instead of barley increased OM digestibility. Also, Salwa et al. (2000), Falcão-e-Cunha et al. (2004), and Sarhan (2005) noticed that inclusion of agriculture by-products in rabbit rations improved $\mathrm{EE}$ digestibility. Also, Omer and Badr (2013) found that $\mathrm{OM}, \mathrm{CF}, \mathrm{EE}$, and NFE digestibilities were significantly $(P$ $<0.05)$ improved with replacement Berseem hay by pea
Table 7 Digestive tract weights and length of the experimental groups

\begin{tabular}{llllll}
\hline Item & $\mathrm{BH}\left(\mathrm{R}_{1}\right)$ & $\mathrm{MBH}\left(\mathrm{R}_{2}\right)$ & $\mathrm{SBVH}\left(\mathrm{R}_{3}\right)$ & $\mathrm{PVH}\left(\mathrm{R}_{4}\right)$ & $\mathrm{SEM}$ \\
\hline $\begin{array}{l}\text { Slaughter weight (SW), g } \\
2451\end{array}$ & 2188 & 2353 & 2169 & 91.94 \\
Stomach (g or \% of SW) & & & & & \\
Full & 100 & 86 & 93 & 79 & 5.26 \\
& 4.08 & 3.93 & 3.95 & 3.64 & 0.21 \\
Empty & 27 & 25 & 28 & 23 & 1.25 \\
& 1.10 & 1.14 & 1.19 & 1.06 & 0.05 \\
Content & 73 & 61 & 65 & 56 & 5.34 \\
& 2.98 & 2.79 & 2.76 & 2.58 & 0.22 \\
Small intestine (g or \% of SW) & & & & \\
Full & 119 & 83 & 86 & 100 & 6.59 \\
& $4.86^{\mathrm{a}}$ & $3.79^{\mathrm{b}}$ & $3.65^{\mathrm{b}}$ & $4.61^{\mathrm{ab}}$ & 0.21 \\
Empty & $71^{\mathrm{ab}}$ & $54^{\mathrm{b}}$ & $59^{\mathrm{b}}$ & $81^{\mathrm{a}}$ & 4.21 \\
& $2.90^{\mathrm{ab}}$ & $2.47^{\mathrm{b}}$ & $2.51^{\mathrm{b}}$ & $3.73^{\mathrm{a}}$ & 0.21 \\
Content & 48 & 29 & 27 & 19 & 5.56 \\
& $1.96^{\mathrm{a}}$ & $1.32^{\mathrm{ab}}$ & $1.15^{\mathrm{ab}}$ & $0.88^{\mathrm{b}}$ & 0.18
\end{tabular}

Large intestine (g or \% of SW)

$\begin{array}{llllll}\text { Full } & 201 & 162 & 197 & 147 & 10.62 \\ & 8.20 & 7.40 & 8.37 & 6.78 & 0.41 \\ \text { Empty } & 68 & 82 & 64 & 78 & 4.59 \\ & 2.77 & 3.75 & 2.72 & 3.60 & 0.26 \\ \text { Content } & 133^{\mathrm{a}} & 80^{\mathrm{ab}} & 133^{\mathrm{a}} & 69^{\mathrm{b}} & 11.96 \\ & 5.43^{\mathrm{ab}} & 3.65^{\mathrm{ab}} & 5.65^{\mathrm{a}} & 3.18^{\mathrm{b}} & 0.47\end{array}$

Total digestive tract ( $\mathrm{g}$ or $\%$ of SW)

$\begin{array}{llllll}\text { Full } & 420 & 331 & 376 & 326 & 18.65 \\ & 17.13 & 15.13 & 15.98 & 15.03 & 0.62 \\ \text { Empty } & 166 & 162 & 151 & 181 & 6.44 \\ & 6.77 & 7.40 & 6.42 & 8.34 & 0.39 \\ \text { Content } & 254^{\mathrm{a}} & 169^{\mathrm{ab}} & 225^{\mathrm{ab}} & 145^{\mathrm{b}} & 18.99 \\ & 10.36^{\mathrm{a}} & 7.73^{\mathrm{ab}} & 9.56^{\mathrm{ab}} & 6.69^{\mathrm{b}} & 0.63\end{array}$

Digestive tract length, $\mathrm{cm}$

$\begin{array}{llllll}\text { Stomach } & 17 & 20 & 18 & 18 & 0.87 \\ \text { Small intestine } & 360 & 341 & 350 & 354 & 11.91 \\ \text { Large intestine } & 90 & 91 & 69 & 79 & 5.08\end{array}$

$\mathrm{a}$ and $\mathrm{b}$ : means in the same row having different superscripts differ significantly $(P<0.05)$

SEM standard error of mean, $B H$ Berseem hay, $M B H$ Mung bean husks, SBVH soybean vein hay, $P V H$ peanut vein hay

straw in rations at different levels of replacement $(0,25$, 75 , and $100 \%$ ). Also, feeding $30 \%$ pea pods hulls diet had better nutritive value compared to control (Sarhan 2005). Meanwhile, Myrie et al. (2008) showed that most feedstuffs contain anti-nutritional factors such as insoluble fibers, ligins, tannins and lectins, intake of these anti-nutritional factors reduced nutrient digestibility and increased endogenous protein losses, through increasing of intestinal mucus secretion.

Data concerning with performance Table 4 in harmony with those reported by Asar et al. (2010), Omer et al. (2011), and Omer and Badr (2013) concluded that inclusion dried faba bean straw, strawberry by-products, pea 
Table 8 Blood parameters of the experimental groups

\begin{tabular}{llllll}
\hline Item & $\mathrm{BH}\left(\mathrm{R}_{1}\right)$ & $\mathrm{MBH}\left(\mathrm{R}_{2}\right)$ & $\mathrm{SBVH}\left(\mathrm{R}_{3}\right)$ & $\mathrm{PVH}\left(\mathrm{R}_{4}\right)$ & $\mathrm{SEM}$ \\
\hline Total protein $(\mathrm{g} / \mathrm{dl})$ & $5.81 \mathrm{~b}^{\mathrm{c}}$ & $5.68^{\mathrm{c}}$ & $6.82^{\mathrm{a}}$ & $6.76^{\mathrm{ab}}$ & 0.20 \\
Albumin (g/ dl) & 4.14 & 3.71 & 3.68 & 4.67 & 0.20 \\
Globulin (g/ dl) & $1.67^{\mathrm{b}}$ & $1.97^{\mathrm{b}}$ & $3.14^{\mathrm{a}}$ & $2.09^{\mathrm{b}}$ & 0.21 \\
Albumin: globulin ratio & $2.48^{\mathrm{a}}$ & $1.88^{\mathrm{ab}}$ & $1.17^{\mathrm{b}}$ & $2.23^{\mathrm{ab}}$ & 0.21 \\
Total lipids (mg/dl) & 282 & 301 & 248 & 367 & 27.15 \\
Triglycerides (mg/dl) & 62.03 & 55.12 & 85.52 & 97.86 & 9.90 \\
Total cholesterol (mg/dl) & 49.79 & 51.35 & 47.49 & 53.74 & 1.16 \\
HDL (mg/dl) & $16.03^{\mathrm{b}}$ & $18.96^{\mathrm{a}}$ & $16.63^{\mathrm{b}}$ & $17.35^{\mathrm{ab}}$ & 0.44 \\
LDL (mg/dl) & 33.76 & 32.39 & 30.86 & 36.39 & 1.13 \\
GPT (U/l) & $77.53^{\mathrm{a}}$ & $76.26^{\mathrm{ab}}$ & $73.63^{\mathrm{b}}$ & $75.11^{\mathrm{ab}}$ & 0.61 \\
GOT (U/l) & 37.79 & 38.55 & 41.70 & 42.38 & 0.95 \\
Creatinine (mg/dl) & 0.98 & 1.04 & 0.75 & 0.97 & 0.06 \\
Alkaline phosphatase & 73.26 & 59.16 & 77.12 & 70.87 & 3.45 \\
(U/l) & & & & & \\
\hline
\end{tabular}

$\mathrm{a}$ and $\mathrm{b}$ : means in the same row having different superscripts differ significantly $(P<0.05)$

SEM standard error of mean, $B H$ Berseem hay, $M B H$ Mung bean husks, SBVH soybean vein hay, $P V H$ peanut vein hay, $H D L$ high-density lipoprotein, $L D L$ low-density lipoprotein

straw, and corn-cob meal can be substituted for dried clover and barley in growing rabbit rations without any adverse effect on their performance.

Also the present results in agreement with those found by García et al. (1993) who showed that incorporation sugar beet pulp up to $15 \%$ did not effect on growth performance. On the other hand, Salwa et al. (2000) established that TBWG of rabbits fed dried watermelon by-product inclusion up to $12 \%$ as replacing for clover hay were not significantly different. In addition, Abo EL-Maaty et al. (2014) noted that rabbits fed rations replaced clover hay with cucumber (Cucumis sativus L.) vines straw up to $75 \%$ resulted in comparable growth performance compared to control. On the other hand, the present results in contrast direction with those noticed by Zeweil (1992) who noted that rabbits received $50 \%$ pea by-product in their diet recorded higher feed intake by $23 \%$ than those fed the control diet. Moreover, Amber et al. (2002) found that feed intake was significantly $(P<0.001)$ increased for rabbits fed Mung bean hay and rice straw compared with those received sugar beet pulp and sweet potato tops. Also, Galal et al. (2014) observed that rabbit received ration containing $40 \%$ strawberry vines by-product replaced from Berseem hay tended to significant $(P<$ 0.05) higher feed intake. Meanwhile, Al-Shanti (2003) noted that insignificant differences in feed intake were noticed when rabbits fed 5 to $20 \%$ inclusion levels of either olive cake or olive pulp.

Results in Table 5 are in harmony with El-Adawy and Borhami (2001), El-Gendy et al. (2002), Abdel-Magid Soha (2005), El-Medany et al. (2008), and Omer et al. (2011) who reported that replacing clover hay by peanut hay, dried sugar beet tops, pea, chick pea, kidney beans straw, dried carrot processing waste, or strawberry by-products in rabbit diets had no significant in dressing percentages. On the other hand, Asar et al. (2010) noticed that rabbits that received diet which contained corn-cob meal with Berseem hay recorded the highest dressing percentage value that reached to $5.48 \%$ over the control. Also, Sarhan (2005) showed that dressing percentages of rabbits fed pea vines hay or pea pods hulls supplemented diets were higher than the control group. However, in contrast, Amber et al. (2002) observed that dressing percentage values were significantly lower for rabbits fed diets which contained sugar beet pulp, sweet potato tops, or Mung bean hay than those fed the control.

Data of external and internal offals (Table 6) in agreement with those reported by Zeweil (1992) and Asar et al. (2010) who observed that there were no significant differences found in the liver, heart, spleen, and kidney relative weights due to dietary inclusion of pea by-product, corn-cob meal, alfalfa hay, Berseem hay, or dried faba bean straw.

The length of the small intestine (Table 7) disagreement with those observed by Asar et al. (2010) who noted that a highly significant increase in the length of small intestine was recorded by rabbits fed diets of corn-cob meal plus Alfalfa hay.

Data of blood parameters (Table 8) in agreement with those obtained by Abdel-Magid Soha (1997), Gad Alla (1997), Mohamed (1999), El Sayed et al. (1999), Tag El-Din et al. (2002), Abdel-Magid Soha (2005), Abou Sekken et al. (2008), and El-Medany et al. (2008). Meanwhile, when $\mathrm{BH}$ was replaced by pea straw at different levels $(0,25,50$, and 100\%), alkaline phosphatase increased $(P<0.05)$, total cholesterol decreased $(P<$ $0.05)$, and total protein and globulin were increased in tested rations (Omer and Badr 2013).

\section{Conclusion}

From the previous study, agriculture by-products such as peanut vein hay, straws of kidney beans, barley, flax, pea, and others may help in the solution of the shortage of animal feeding (Omer et al. 2012; Omer and Badr 2013). Berseem hay that considered as the important source of roughage depending on using it in rabbit ration formulation can be replaced by $50 \%$ of different roughages such as soybean vein hay, peanut vein hay, and Mung bean husks without any adverse effect on their gain, digestion coefficients, carcass characteristics, and blood constituents.

\section{Acknowledgements}

This work was supported by scientific project section, National Research Centre (P11030122) under the title "Increasing productivity of some un-traditional crops and crop residues for animal feeding." 


\section{Funding}

Project No. (P11030122) under the title "Increasing productivity of some un-traditional crops and crop residues for animal feeding." National Research Centre.

\section{Availability of data and materials}

"Not applicable" for that section.

\section{Authors' contributions}

HA-AAO cooperated in the plane of work, field work, chemical analysis, data calculations, statistical analyses of data, and writing of the MS and helped in the publication. MFE K provided experimental animals and facilities. SMA cooperated in the plane of work, field work, and revision of the MS and helped in the publication. SSA-M cooperated in the plane of work and field work and followed the publication with the journal (corresponding author). SIE-N cooperated in the blood sample analysis. BAB contributed in providing facilities. All authors read and approved the final manuscript.

\section{Ethics approval and consent to participate}

"Not applicable" for that section.

\section{Consent for publication}

"Not applicable" for that section.

\section{Competing interests}

The authors declare that they have no competing interests.

\section{Publisher's Note}

Springer Nature remains neutral with regard to jurisdictional claims in published maps and institutional affiliations.

\section{Author details}

${ }^{1}$ Animal Production Department, National Research Centre, 33 El-Bohouth Street, P.O: 12622, Dokki, Cairo, Egypt. ' Field Crops Research Department, National Research Centre, 33 El-Bohouth Street, P.O: 12622, Dokki, Cairo, Egypt.

Received: 3 September 2018 Accepted: 5 November 2018 Published online: 27 November 2018

\section{References}

Abdel-Magid Soha S (1997) Using some agro-industrial by-products in rabbits nutrition. M.Sc. Thesis. Faculty of Agricultural, Cairo, University

Abdel-Magid Soha S (2005) Nutritional studies on leguminous straw in feeding growing rabbits. Ph.D.Thesis. Faculty of Agricultural, Cairo, University

Abdel-Magid Soha S, Abd El-Rahman HH, Mohamed MI, Awadalla IM (2008) Utilization of chick pea straw and pea straw in feeding growing Rahmani lambs. American-Eurasian J Agric Environ Sci 4(2):214-217

Abou Sekken MS, Fahmy AA, Ahmed SS (2008) Performance of growing rabbits fed diets containing different levels of Fennel and Marjoram waste under desert environmental conditions. Egyptian J Nutrition Feeds 11(1):1-23

Abou-Akkada AR (1988) For national strategic for increasing feedstuff in Egypt. 1st National Conf. On Role Of Scientific Research in Developing Animal Health, Academy of Scientific Research and Technology, 25-29 September . Cairo Egypt (In Arabic)

Abou-Raya AK (1967) Animal and Poultry Nutrition 1st Ed. Pub. Dar El-Maarif, Cairo (Arabic text book)

Allain CC, Chan PCS, Richmond W, Fu PC (1974) Enzymatic determination of total serum cholesterol. Clin Chem 20:470-475

Al-Shanti HA (2003) Effect of feeding olive cake on growth performance, nutrients digestibility, carcass traits and blood constituents of growing rabbits. Egypt J Rabbit Sci 13(2):103-116

Amber KH, Isshak NS, El-Abd Niamat M (2002) Effect of dietary fiber source on performance of growing New Zealand White rabbits. Egypt Poult Sci 22:127-146

AOAC (2005) Official Methods of Analysis, 18th edn. Association of official analytical chemists, Washington, DC

Armstrong WD, Carr CW (1964) Physiological Chemistry: Laboratory directions 3: 75. Buger Puplishing Co, Minneapolis

Asar MA, Mona O, Yakout HM, Safoat A (2010) Utilization of corn-cob meal and faba bean straw in growing rabbits diets and their effects on performance, digestibility and economical efficiency. Egypt Poult Sci 30(II):415-442
Assmann G (1979) HDL-cholesterol precipitant. In: Internist, vol 20. Randox Labs. Ltd. Crumlin Co, Antrim, pp 559-567

Beliefield A, Goldberg DM (1971) Estimation of serum alkaline phosphates. Enzyme 12:561

Blasco A, Quhayaun J, Masoscro G (1993) Hormanization of criteria and terminology in rabbit meat research. World Rabbits Sci 1:3-10

Blaxter KL (1968) The energy metabolism of ruminants, 2nd edn. Charles Thomas Publisher, Spring field

Calsamiglia S, Stem MD, Frinkins JL (1995) Effects of protein source on nitrogen metabolism in continuous culture and intestinal digestion in vitro. J Anim Sci 73:1819

Cheeke PR (1987) Rabbit Feeding and Nutrition. Academic Press, Orlando

Cheeke PR (1986) Potential of rabbit production in tropical and subtropical agricultural system. J Anim Sci 63:1581-1586

Cheeke PR, Patton NM, Lukefahr SD, Mcnitt JI (1987) Rabbit production. The Interstate printers and Publishers Inc., Danville

Doumas BL, Watson TWA, Biggs T (1971) Albumin standards and measurement of serum with bromocresol green. Clin Chem Acta 31:87

Duncan DB (1955) Multiple Rang and Multiple F-Test Biometrics, vol 11, pp 1-42

El Sayed IA, Abdellah GA, Mervat B, Khattaby SE (1999) Effect of inclusion pea hulls and seaweeds (Polysiphania spp.) in diets of growing rabbits on their performance and blood constituents. Egypt Poultry Sci 19:141-159

El-Adawy MM, Borhami BE (2001) Utilization of peanut hay and dried sugar beet tops in feeding of growing rabbits. Egypt J Nutr Feeds 4(Special Issue):869-883

El-Gendy KM, Abd EL-Baki SM, Sarhan MA, Moawd RI (2002) Evaluation of sweet Lupin (Lupin albus) as green forage for sheep and rabbits $3^{\text {rd }}$ Sci. Congr., Rabbit Production in Hot Climates; 8-11 October, 677-692

EL-Maaty H, Abo MA, EL-Samra HA, Egla A, Qota EM, EL-Desouky Sheren M (2014) Performance and economical efficiency of growing New Zealand White rabbits fed cucumber (cucumis sativus I) veins straw without or with some feed additives under Egyptian conditions. Egypt Poultry Science 34(2):413-431

El-Medany NM, Hashem NA, Abdl-Azeem F (2008) Effect of incorporating dried carrot processing waste in growing rabbit diets. Egyptian J Nutr Feeds 11(1):25-37

EMA. Egyptian Ministry of Agricultural, Statistical Yearling Book. Ministry of Agricultural, Cairo (In Arabic). 2003

Falcão-e-Cunha L, Helena P, Freire JPB, Castro Solla L (2004) Effects of Alfalfa hay, wheat bran or beet pulp, with or without sunflower oil, on caecal fermentation and on digestibility in the rabbit. Anim Feed Sci Technol 117:131-149

Fossati P, Prencipe L (1982) Serum Triglycerides Determined Colorimetrically with an Enzyme that Produces Hydrogen Peroxide. Clin Chem, 28, 2077-2080

Gad Alla SAZ (1997) Utilization of some agricultural by-products in feeding rabbits. Ph.D. Thesis, Fac. Agric., Kafr El-Sheikh, Tanta Univ., Egypt

Galal HMF, El Menniawy MA, Abo-Fadel MH, Khir AA, Abdel-Azeam SN (2014) Some nutritional studies on using strawberry (Fragaria $x$ Ananas) vine as hay in rabbit ration. J Animal Poultry Prod Mansoura Univ 5(12):635-647

García G, Galvez JF, De Blas JC (1993) Effect of substitution of sugar beet pulp for barley in diets for finishing rabbits on growth performance and energy and nitrogen efficiency. J Anim Sci 71:1823-1830

Ghazalah AA, El-Shahat AA (1994) Digestibility and acceptability of some agroindustrial by-products by rabbits. Egypt Poultry Science 14:401-424

Goering HK, Van Soest PJ (1970) Forge fiber analysis (apparatus, reagents, procedure and some applications). Agric. Hand book 379, USDA Washington, and DC., USA

Harold V (1975) Colorimetric determination of glutamate pyruvate and oxaloacetic transaminase, practical Clin Biochem, 4th edn, p 294

Husdan H (1968) Chemical determination of creatinine with deproteinization. Clin Chem 14:222

McNamara JR, Cohn JS, Wilson PW, Schaefer EJ (1990) Calculated values for lowdensity lipoprotein cholesterol in the assessment of lipid abnormalities and coronary disease risk. Clin Chem 36:36-42

Mohamed SA (1999) Digestibility and acceptability of some agricultural by-products by growing rabbits. M.Sc. Thesis. Faculty of Agricultural, Cairo, University

Myrie SB, Bertolo RF, Sauer WC, Ball RO (2008) Effect of common antinutritive factors and fibrous feedstuffs in pig diets on amino acid digestibilities with special emphasis on threonine. J Anim Sci 86:609-619

NRC (1977) National Research Council. Nutrient requirements of rabbits. National Academy of Science, Washington, D.C

Omer HAA, Ali FAF, ShAM I (2011) Strawberny by-products as a partial replacement of clover hay in rabbit diets. American-Eurasian J Agric Environ Sci 11(6):815-823

Omer HAA, Badr AMM (2013) Growth performance of New Zealand White rabbits fed diets containing different levels of pea straw. Life Sci J 2(10):1815-1822 
Omer HAA, Tawila MA, Gad Sawsan M (2012) Feed and water consumptions, digestion coefficients, nitrogen balance and some rumen fluid parameters of Ossimi sheep fed diets containing different sources of roughages. Life Sci J 9(3):805-816

Pisani T, Gebski CP, Leary ET (1995) Accurate direct determination of low- density lipoprotein, cholesterol assay. Arch Pathol Lab Med 119:1127

Postma T, Stroes JA (1968) Lipids screening in clinical chemistry. Clin Chim Acta 22:569

Reitman S, Frankel S (1957) Calorimetric method for the determination of serum glutamic oxaloacetic and glutamic pyruvate transaminase. An J Clin Path 28:56

Sadek Enath E (2013) Economic and environmental impacts of using agricultural waste for producing non-traditional feeds. Proceeding of the $14^{\text {th }}$ Scientific Conference of Animal Nutrition, 26-29 November, 2013. Hurghada, Egypt. Egyptian J Nutr Feeds 16((2) Special Issue):139-147

Salwa, GKG, Radwan MSM, Abo Zeid AE, Zanaty G.A (2000) Feeding rabbits dried watermelon by-product (Citrullus lanatus Var.) as a replacing feed for clover hay. $8^{\text {th }}$ Agricultural Development Research Conference; November, 20-22, Faculty of Agriculture, Ain Shams University, Egypt

Sarhan MA (2005) Utilization of agro-industrial by-products of pea (Pisum Sativum) in growing rabbit diets. Egypt J Rabbit Sci 15(2):157-172

SPSS (2008) Statistical package for Social Sciences, Statistics for Windows, Version 17.0. Released 2008. SPSS Inc., Chicago

Tag El-Din TH, Al-Samra HA, Ismail FS, Samy SS (2002) Effect of using graded levels of Phaseolus vulgaris straw in growing rabbit diets. $3^{\text {rd }}$ Sci. Congr., Rabbit Production in Hot Climates; 8-11 October, 643-659

Tietz NW (1986) A method for the rapid determination of albumin of blood plasma. P.589 in textbook of clinical chemistry. W.B Saunders Company, Philadelphia

Van Soest PJ, Robertson JB, Lewis BA (1991) Methods for dietary fiber, neutral detergent fiber and non starch polysaccharides in relation to animal performance. J Dairy Sci 74:3583-3597

Witt I, Trendelenburg C (1982) A method for the rapid determination of total protein plasma. J Clin Biochem 20:235

Zeweil HS (1992) Use of a residue from pea (Pisum Sativum) processing in feeding growing rabbits. Egypt Poultry Sci 12:17-30

\section{Submit your manuscript to a SpringerOpen ${ }^{\circ}$ journal and benefit from:}

- Convenient online submission

- Rigorous peer review

- Open access: articles freely available online

- High visibility within the field

- Retaining the copyright to your article

Submit your next manuscript at $\boldsymbol{\nabla}$ springeropen.com 